\title{
PERSPECTIVA INTERNACIONAL ACERCADE INDICADORES DE QUALIDADE EM CURSOS DE DOUTORADO EM ENFERMAGEM ${ }^{1}$
}

\author{
SETTING THE STAGE: INTERNATIONAL PERSPECTIVES ON QUALITY \\ INDICATORS FOR DOCTORAL NURSING EDUCATION \\ PERSPECTIVA INTERNACIONALEN EL ÁMBITO DE INDICADORES DE \\ CALIDAD EN CURSOS DE DOCTORADO EN ENFERMERIA
}

Eloita Neves Arruda ${ }^{2}$

Alcione Leite da Silva ${ }^{3}$

\begin{abstract}
RESUMO: As autoras comparam/contrastam os indicadores de qualidade para programas de doutorado em três paises, Estados Unidos, Brazil e Venezuela, indicando diferenças e semelhanças e apontando questões que possam globalmente afetar a educação de doutorado em enfermagem. Tal análise é baseada em três documentos: o da Associação Americana das Escolas de Enfermagem - AACN (1993); o da CAPES - Brasil (1998) e o do Conselho Nacional Consultivo de Pós-Graduação da Venezuela (1987). As autoras utilizaram como referencial os conceitos de qualidade formal e qualidade politica (Demo, 1994) e as dimensões sociopolitica, técnica e subjetiva de López e Calderon (1996). Os indicadores são distribuidos dentre os seguintes componentes: corpo docente, programa de estudos, recursos, pesquisa e estudantes. Finalmente, as autoras discutem indicadores em relação ao plano para avaliação de qualidade de programas de doutorado em enfermagem.
\end{abstract}

PALAVRAS-CHAVE: educação de doutorado, garantia de qualidade, educação em enfermagem

\section{INTRODUÇÄO}

A complexidade crescente da vida em sociedade, aliada a ampliação dos sistemas de comunicação mundial, vem desafiando, nas últimas décadas, os cursos de doutorado, no sentido de buscarem continuamente aprimorar os recursos e processos para assegurar a qualidade destes. O processo de globalização da economia internacional emerge com implicações significativas nos sistemas de relações humanas, nas demandas de tecnologias, bem como nos indicadores de qualidade do processo de formação profissional/disciplinar, com repercussões nos serviços prestados à sociedade. Neste sentido, um desafio a ser enfrentado é o de buscarmos uma visão mais global para entender com percepção técnica e sensibilidade social as assimetrias criadas por um mundo que se globaliza de forma heterogênea, criando uniões e cisões em todos os niveis, alianças e rupturas, que exigem novos instrumentos de análise e grande

' Conferência proferida no Encontro International Network of Doctoral Education - Quality in Doctoral Education: Empowerment Through Nursing Science. Londres, 27 Junho de 1999.

${ }^{2}$ Enfermeira, Doutora em Ciências da Enfermagem. Professora Titular Aposentada Participante do Programa de Pós Graduação Enfermagem UFSC. Coordenadora do Programa Integrado de Pesquisa Cuidando e Confortando (PIP C\&C).

${ }^{3}$ Enfermeira, Doutora em Filosofia da Enfermagem, Professora Titular UFSC, Coordenadora didático-pedagógica do Curso de Doutorado em Enfermagem da UFSC, membro do PIP C\&C, Coordenadora do Núcleo de Pesquisa "Cuidando e Confortando: Tecnologias Pró Ser e Viver Saudável - UFSC" 
percepção para serem entendidas em toda a sua profundidade. Neste cenário, o futuro dos cursos de doutorado estará sendo influenciado não só pelas necessidades da sociedade, da profissão e pelas tendências dos sistemas de saúde, conforme apontou Meleis (1988), mas também pela capacidade de estabelecermos parcerias no âmbito local, regional, nacional e internacional, respeitando as diferenças culturais, a cosmovisão. Contudo, este processo de articulação implica necessariamente em considerarmos a capacidade de integração grupal/ local, ou seja, a capacidade reciproca dos membros em se identificar com os interesses, valores e metas do grupo profissional.

Nesta conjuntura, faz-se necessário repensar os indicadores de qualidade da educação no doutorado em enfermagem, como um dos requisitos imprescindiveis para a integração local e para a busca de possibilidades de articulações tanto no âmbito local e global. Ao nos propormos, neste trabalho, a analisar os indicadores de qualidade para programas de doutorado em enfermagem em três paises, Estados Unidos, Brasil e Venezuela, apontamos diferenças e semelhanças, bem como identificamos questões que poderão afetar globalmente a educação nos cursos de doutorado de enfermagem. A escolha destes países, além do Brasil, deu-se por solicitação de uma análise comparativa por parte das organizadoras do encontro e face aos seguintes motivos: 1) a experiência de ambas as autoras em ensino de pós graduação em enfermagem no Brasil e a experiência da primeira em avaliação de cursos de pós graduação em Comissão de Consultores da CAPES ${ }^{4} ; 2$ ) a realização do doutorado e pós-doutorado da primeira autora nos Estados Unidos e de doutorado sanduíche ${ }^{5}$ da segunda autora neste mesmo país; 3) a participação da primeira autora na implantação, na qualidade de docente, do primeiro doutorado em enfermagem, recentemente criado em pais da América do Sul, de fala espanhola.

São três realidades diferentes em seus aspectos culturais, sociais, políticos, econômicos e geográficos, que se refletem no processo de vida e, conseqüentemente, de resolução de seus problemas internos e no estabelecimento de relações internacionais. Nestes paises, a enfermagem vem historicamente construindo, de forma diversificada, a sua inserção profissional na sociedade, o seu desenvolvimento como disciplina e sua tradição em cursos de doutorado. No que se refere a esta tradição, a história da formação de enfermeiras em nivel de doutorado, nos Estados Unidos, evidencia três estágios, iniciando pelo doutorado em educação - EdD (1924-1959), passando pelo doutorado obtido em outras áreas do conhecimento (1960-1969) e finalmente, implementando diferentes modalidades de doutorado em enfermagem ( $P$ hD, DNS, ND) ${ }^{6}$, a partir dos anos 70 (Grace, 1978; Carpenter, 1996), havendo até 1993, 54 cursos (Gemain et al., 1994). No Brasil, esta tradição é ainda mais recente, tendo em vista que o primeiro curso de doutorado em enfermagem foi criado em 1982, contando até o momento com sete cursos em funcionamento. Na Venezuela, foi criado o primeiro curso em abril de $1998 \mathrm{em}$ modalidade de rede entre as Escolas de Enfermagem do Pais e cooperação internacional com a UFSC. Estudos para a implantação de outros cursos em países da América Latina estão sendo efetuados com base em parcerias inter-institucionais, com cooperação internacional do Brasil, através de cursos já consolidados e considerados de alta qualidade. Assim, os desafios impõem aos/às enfermeiros/as um processo cooperativo, solidário, para fazer face às mudanças do cenário de atuação das instituiçōes de ensino na sociedade. Tais iniciativas demonstram que a palavra de ordem é articular forças e diluir as fraquezas para sobreviver e avançar, perseguindo ideais

\footnotetext{
4 Esta comissão é constituida por docentes de enfermagem, consultores, que avaliam a cada dois anos os cursos de pós graduação - mestrado e doutorado no pais.

${ }^{5}$ Designação dada a modalidade de bolsa oferecida pelo governo brasileiro (CAPES e CNPq) aos alunos de doutorado para desenvolverem durante periodo não superior a um ano, parte de sua formaçăo acadêmica em instituição congẻnere no exterior.

- Estas diferentes modalidades de doutorado são discutidas na literatura por Meleis (1988). SnyderHalpern(1986), Murphy (1981).
} 
eticamente sustentáveis para assegurar o oferecimento de cursos que atendam os indicadores que atendam os indicadores locais e globais de qualidade em um mercado complexo e competitivo, determinado pelo processo de internacionalização/globalização.

\section{REFERENCIAL TEÓRICO - INDICADORES DE QUALIDADE}

Pensar em indicadores de qualidade implica em, primeiramente, explicitar algumas concepções acerca de conceito de qualidade, buscando ancoragem na educação. O conceito de qualidade está associado às caracteristicas ou atributos essenciais do grau de excelência (Davies, 1976). O conceito de qualidade tem evoluído de forma lenta e gradual, passando pela noção de controle de qualidade, para chegar aos nossos dias como cultura de qualidade, para definir uma participação coletiva, solidária e diferenciada, com vistas a conquistar a excelência dos resultados (Drugg, Ortiz, 1994).

Demo (1994) distingue duas dimensões no conceito de qualidade, ou seja, qualidade formal e qualidade politica. A qualidade formal significa a habilidade de manejar meios, instrumentos, formas, técnicas, procedimentos, diante dos desafios do desenvolvimento. Entre eles ressaltam manejo e produção do conhecimento. A qualidade politica, por sua vez, refere-se à competência do sujeito em termos de se fazer e de fazer história, diante dos fins da sociedade humana. É condição básica da participação. Dirige-se a fins, valores e conteúdos. Neste sentido, conhecimento sem qualidade politica perde a noção da ética e serve a qualquer ideologia. Assim, temos de um lado a arma mais potente de inovação, comandada pelos avanços sistemáticos do conhecimento. De outro, temos o desafio permanente de discutir, rever e refazer o sentido histórico da inovação ou de humanizar o progresso (Demo, 1994).

Para este mesmo autor, tanto o conhecimento quanto a educação são obra humana e por isso Ihe cabe o desafio da qualidade. Enquanto, na qualidade formal trata-se da arte de descobrir, na qualidade politica trata-se da arte de fazer. Assim, a garantia de qualidade centrase no desafio de manejar os instrumentos adequados para fazer a história humana.

A qualidade formal do conhecimento e da educação é instrumento primordial de inovação. Como meio, orienta-se pelo fim, que é a qualidade politica. A prática da qualidade refere-se, pois, à competência inovadora e humanizadora de um sujeito histórico, formalmente preparado para manejar e produzir conhecimento, enquanto força primordial, que decide considerar, mais do que outros fatores, a cidadania e a competência construtiva e participativa (Demo,1994).

Em se tratando da avaliação de qualidade da educação superior em enfermagem, esta é entendida por nós a partir de López e Calderon (1996), como um processo de análise permanente da situação sócio-epidemiológica, política, econômica, cultural, das práticas profissionais de enfermagem e do mercado de trabalho em saúde, para ajustar o processo de educação superior em enfermagem, possibilitando sua legitimidade e pertinência social, na medida em que responda às necessidades do contexto no qual egressem profissionais técnica, científica, humana $\mathrm{e}$ politicamente qualificados.

Estas autoras (López e Calderon, 1996) avançam na proposta de Demo (1994), ao proporem para a avaliação da educação superior em enfermagem, três dimensões fortemente conectadas entre si: as dimensões sócio-politica, técnica e subjetiva. A dimensão sócio - politica se refere às demandas exógenas ao sistema educativo contidas na finalidade da educação em enfermagem: a de produzir, reproduzir e transmitir conhecimentos socialmente válidos. Além disto, esta dimensão atende às exigências especificas do sistema cultural referentes ao desenvolvimento da identidade nacional e do desenvolvimento de valores, atitudes e condutas que tornem possivel o funcionamento de uma sociedade democrática, solidária e participativa. Também incluem nesta dimensão as demandas do sistema econômico no sentido de desenvolver capacidades para a incorporação do egresso ao mundo produtivo, oferecendo aporte científico para seu desenvolvimento, comunicando-se efetivamente, trabalhando em equipe exercendo 
a função produtiva de maneira crítica e criadora.

A dimensão técnica do sistema educativo diz respeito às opçöes técnicas que modelam a forma como se organiza a educação para atender às demandas da sociedade, dentre elas: a organização da proposta educativa, a intervenção didática para produção e transmissão de conhecimentos e valores éticos, pessoais e profissionais; os processos institucionais - clima organizacional, estratégias de avaliação, modelos de distribuição do tempo, espaço e poder que possibilitem discussões abertas em convivio com a diversidade intelectual e de ideologias.

A dimensão subjetiva se refere aos componentes do dominio afetivo no processo educativo, expressos por sentimentos do/a educador/a e do/a educando/a em relação à: sentirse competente, satisfeito e motivado para o desempenho do papel, em seu ambiente social e profissional.

Conseqüentemente, ao adotarmos a visão de Demo (1994), e López e Caderón (1996), fica claro que não podemos restringir qualidade a meros procedimentos formais ou a mero conhecimento, tendo em vista que a educação não se reduz ao conhecimento. Deste modo, ao buscarmos a qualidade formal, política e subjetiva na educação, teremos que articular teoria e prática, procurando não apenas instrumentalizar-nos, mas, sobretudo, atuar na realidade, de forma inovadora, para transformá-la.

Pelo exposto, podemos afirmar que a avaliação da qualidade da educação nos cursos de doutorado em enfermagem consiste em um processo realizado com competência inovadora e humanizadora, construído historicamente e de forma participativa por sujeitos que produzem conhecimento (educadores, educando e administradores) e por sujeitos representantes dos segmentos que consomem os produtos e os serviços que oferecemos à sociedade (educando, empregadores e órgão de fomento). Esta avaliação deve favorecer e apontar caminhos para uma educação que forme cidadãos criticos e criativos, capazes de indicar o rumo histórico, coletivamente pretendido. Além disto, um curso de excelência atrairá tanto educadores, quanto educandos altamente qualificados. Nesta perspectiva, eles poderão contribuir para fortalecer a disciplina de enfermagem e ampliar a sua participação com as outras disciplinas, com vistas a solução criativa e efetiva de problemas da sociedade, para a complexidade crescente de qualidade no processo de ser e viver saudável.

Um processo de avaliação dos cursos de doutorado em enfermagem terá que definir indicadores de qualidade apropriados. Esta definição constitui-se em espaço de decisão ética e política, que não deve ser abandonado quando se trata de conquistar um direito inalienável à qualidade da educação. A conquista da qualidade se constitui em um dos desafios que nos deparamos, principalmente quando sofremos inúmeros tipos de restrição e segmentação de caráter mercantil.

Outro desafio refere-se à impossibilidade de homogeneizar ou estabelecer um padrão unificado ou universal de indicadores de qualidade para os cursos de doutorado em enfermagem. Isto porque, a qualidade da educação se define através das necessidades de cada sociedade em um espaço e tempo definido, sendo também determinada pela situação sócio-cultural, politica e econômica dos paises, regiões e comunidades locais. Neste sentido, a busca de articulação entre cursos de doutorado perpassa pela definição de indicadores globais/ internacionais, levando em conta que os indicadores locais/nacionais são definidos respeitandose as especificidades culturais, políticas e econômicas da realidade local. Há que se considerar também o processo de transformações muito rápidas a que estão submetidas as sociedades, exigindo inovação, criatividade e permanente atualização no estabelecimento dos indicadores de qualidade dos cursos de doutorado em enfermagem.

\section{ANÁLISE DOS INDICADORES DE QUALIDADE DOS CURSOS DE DOUTORADO}

Para analisar os indicadores de qualidade dos três paises em relação aos cursos de 
doutorado, nos servimos dos seguintes documentos: o da Amerin Association of Colleges of Nursing - AACN (1993); o da Coordenação de Aperfeiçoamento de Pessoal de Ensino Superior - Ministério da Educação - CAPES (1998) e; o do Conselho Nacional e Universidades / Conselho Consultivo Nacional de Estudios para Graduados - CCNEPG (1987). O primeiro documento estabelece indicadores para os programas de doutorado em enfermagem (PhD, DNS, EdD) nos Estados Unidos; o segundo trata de uma proposta preliminar de indicadores de um perfil de excelência dos cursos da área de saúde e de enfermagem, sem estabelecer especificidades para cursos de doutorado e; o terceiro aponta indicadores para programas de pós-graduação, especificando, em separado, os indicadores de qualidade para os cursos de mestrado e de doutorado nas áreas de Ciências Naturais, Engenharia e Ciências Sociais.

É interessante destacar que os indicadores da AACN foram elaborados pela enfermeiras para atender às necessidades do desenvolvimento da enfermagem. por iniciativa das Escolas, os do CCNEPG foram estabelecidos pelo Conselho de Universidades com a finalidade de autorização e credenciamento dos cursos, enquanto que os da CAPES foram criados por iniciativa govemamental, com a finalidade de monitorar a distribuição de recursos para os cursos de pós-graduação das diferentes áreas e, atualmente, por delegação do Ministério de Educação, servem como parâmetros para autorização e credenciamento dos cursos.

A partir da análise preliminar dos documentos, adotamos os cinco componentes utilizados para o doutorado em enfermagem da $A A C N$, que são: corpo docente, programa de estudo/ estrutura curricular, recursos, pesquisa e estudantes. A seguir, distribuimos nestes componentes os respectivos indicadores, estabelecendo uma análise comparativa entre os diferentes documentos dos paises indicados.

\section{CORPO DOCENTE}

No que diz respeito ao corpo docente, os três documentos explicitam indicadores que consideram como requisitos que os docentes sejam doutores/pesquisadores na área do curso, que apresentem uma diversidade de perspectiva intelectual e que sejam capazes de conceber e implementar programas de pesquisa, mantendo projetos em andamento, com o engajamento de estudantes. Merece destaque o enfoque dado pela AACN ao afirmar "que o programa de pesquisa e a scholarship continuam ao longo do tempo e se constróem sobre trabalhos anteriores" (1993), tendo em vista que este constitui um indicador importante no desenvolvimento da disciplina de enfermagem.

Os cursos dos paises da América Latina valorizam a experiência anterior do docente do doutorado em orientação e ensino de pós-graduação e a interdisciplinaridade, ou seja, a participação no corpo docente de intelectuais de outras áreas do conhecimento. Também consideram como indicador de qualidade a presença de professor visitante da área e outras formas de participação externa, promovendo o intercâmbio e a cooperação local, regional, nacional e internacional. Explicitam a necessidade da continuidade da formação do docente em cursos de atualização e de pós-doutorado.

Ressaltamos que o Brasil atribui importância ao indicador corpo docente estável e permanente, com dedicação integral ao curso, devendo este, além das atividades de pesquisa, ter envolvimento regular em atividades de ensino e orientação em cursos de pós-graduação e graduação. Os docentes devem ter publicações e apresentar trabalhos em eventos científicos nacionais e internacionais, com qualidade e quantidade estabelecidas, bem como demonstrar projeção nacional, através da participação em comissões, em nivel nacional e intemacional e da participação em conselho editorial de periódicos.

Um dos indicadores considerados no documento da AACN e não contemplados pelos demais refere-se à criação pelos docentes de um ambiente, no qual se evidencia a orientação, a socialização dos estudantes e a existência de uma comunidade de acadêmicos. Os 
indicadores relativos ao corpo docente, acima apresentados, evidenciam uma supremacia da dimensão de qualidade formal/técnica, em detrimento da política/sócio-política. Cabe destacar que o documento da AACN contempla em dois de seus indicadores de corpo docente a dimensão política, estando estas ausentes nos demais documentos. Nenhum dos documentos contempla a dimensão de qualidade subjetiva.

Constatamos a necessidade da inclusão de indicadores que contemplem aspectos na dimensão politica/sócio-politica relacionados a atitudes e comportamentos do docente, no sentido de garantir a qualidade do curso de doutorado. Destacamos a capacidade do docente de conhecer e influenciar nas politicas de pós-graduação e de investigação, contribuindo para o avanço da disciplina de enfermagem. Incluímos também a abertura ao novo, a capacidade de avançar o conhecimento de forma criativa, com coragem para ousar, inovar e posicionar-se, no sentido de comprometer-se a alcançar maior capacidade resolutiva para a melhoria do processo de vida em sociedade. Estes aspectos nos remetem também à dimensão subjetiva, a qual envolve motivação dos diferentes atores para empreender mudanças e alcançar metas; sentimento de satisfação dos docentes com as atividades próprias do sistema educativo e o sentido de domínio da pessoa com o papel que desempenha.

\section{PROGRAMA DE ESTUDO/ESTRUTURA CURRICULAR}

Em se tratando do segundo componente, programa de estudo/estrutura curricular, é comum aos três documentos que as atividades de formação sejam estabelecidas conforme a área de expertise/scholarship das/os docentes, com uma perspectiva pedagógica bem fundamentada, conforme as características do curso e que sejam consistentes com a missão e objetivos do curso e área de concentração. Os documentos da CAPES e CCNEPG, embora explicitem como indicador de qualidade a existência de uma base epistemológica e a formação metodológica para a pesquisa, correspondentes a área específica do curso, deixam de considerar aqueles aspectos do programa de estudos/estrutura curricular referentes ao desenvolvimento do conhecimento e liderança em enfermagem, bem como estratégias analiticas e de liderança para lidar com as questões sociais, éticas, culturais, econômicas e políticas, relacionadas à enfermagem, cuidado da saúde e pesquisa, como o faz o do AACN.

Ainda em relação ao programa de estudos/estrutura curricular, notamos a ênfase da AACN nos seguintes aspectos: identificação de um conteúdo central; oportunidade para os estudantes aprofundarem experiências anteriores para o desenvolvimento de seu papel profissional e; clareza dos requisitos e seqüência destes na progressão dos estudos dos estudantes.

Os três documentos abrangem a dimensão formal/técnica, contudo o da AACN avança no sentido de assegurar o conhecimento necessário para a inserção e a atuação política do/a doutor/a em enfermagem na sociedade. Outro indicador ausente e que nos parece de fundamental importância em um mundo globalizado, diz respeito a flexibilidade da estrutura curricular, com possibilidades de favorecer intercâmbios de docentes e estudantes entre os cursos de doutorado em enfermagem, no âmbito local, regional, nacional e internacional. Deste modo, além do enriquecimento em múltiplas perspectivas culturais, em nivel pessoal/profissional, os intercâmbios poderão favorecer a cooperação e a reciprocidade, através da articulação de forças e diluição das fraquezas entre os cooperados.

Outro indicador que consideramos importante refere-se à dimensão de qualidade subjetiva, acerca da perspectiva ontológica da educação, na medida em que esta possa privilegian a compreensão e desenvolvimento dos seres envolvidos no processo educativo.

\section{RECURSOS/INFRAESTRUTURA}

Com relação ao terceiro componente, ou seja, os recursos / infraestrutura, os documentos 
indicam que os cursos devem possuir instalações física, bibliotecas, apoio técnico e recursos de informática de fácil acesso para professores/as e alunos/as, e receber apoio financeiro e técnico de órgãos de fomento e da própria universidade para o desenvolvimento dos programas de pesquisa.

Estes indicadores são de natureza formal/técnica e não privilegiam as dimensões política/ sócio-política e subjetiva. Em nosso entendimento estes indicadores não são suficientes para assegurar a qualidade, é preciso incluir outros que possibilitem avaliar a autonomia do curso para buscar e administrar seus recursos, bem como para aprimorar a "utilização do processo administrativo na gestão do sistema educativo" (López e Caderón, 1996, p. 06). Da mesma forma, é necessário considerar na avaliação deste componente, a dimensão subjetiva, incluindo indicadores que reflitam o sentimento de satisfação e motivação dos diferentes atores para buscar recursos que continuamente promovam a melhoria da infra-estrutura.

\section{PESQUISA}

Considerando o quarto componente, pesquisa, os documentos apontam como indicadores de qualidade a construção dos programas de pesquisa sobre trabalhos anteriores, contando com apoio de órgãos de fomento. No entanto, o documento da AACN explicita que os programas devem ser congruentes com as prioridades de pesquisa na área e nas comunidades. Já os outros dois documentos enfatizam a necessidade de recursos humanos apropriados para o desenvolvimento de tais programas, incluindo a participação docente e discente. Os documentos da AACN e CAPES estabelecem uma variedade de mecanismos para assegurar a elevada qualidade da pesquisa, tais como, orientação, bancas de qualificação e de defesa, consultoria. O documento da CAPES inclui como indicadores a adequação, abrangência e vínculo entre linhas e projetos de pesquisa em relação às áreas de concentração do curso. Ainda nesta perspectiva, considera a quantidade de projetos em relação à dimensão e qualificação do corpo docente e a participação do corpo discente nos projetos de pesquisa.

Os indicadores, acima mencionados, estão inseridos nas dimensões formal/técnica e política/sócio-política não contemplando a dimensão subjetiva, ou seja, o sentimento em relação à sua competência, satisfação e motivação para realizar e coordenar programas/grupos de pesquisa.

Em se tratando da dimensão política/sócio-politica, achamos fundamental o estabelecimento de indicadores que avaliem a capacidade dos programas/grupos de pesquisa em se articularem, através de uma agenda colaborativa de pesquisas que contemple temáticas prioritárias e comuns entre os pares, em nivel local, regional, nacional e internacional. Nesta perspectiva, temos de considerar que a pesquisa não pode se constituir em um fim em si mesma, mas ter aderência com as reais necessidades da sociedade.

\section{ESTUDANTES}

Em se tratando do quinto componente, estudantes, ressaltamos não haver concordância explícita entre os indicadores dos três documentos. Contudo, em relação ao tempo para a obtenção do título, os três apontam para este indicador, seja reconhecendo a necessidade de definir requisitos para a permanência no curso, seja estabelecendo o tempo máximo de quatro anos, seja requerendo compromisso e dedicação do estudante no curso para que possa completar seus estudos no tempo estabelecido. Por outro lado, o documento da CAPES aponta para indicadores que consideram a adequação entre o número de estudantes e o de docentes, exigindo uma percentagem de titulados em relação à de estudantes admitidos no ano. $O$ documento da AACN destaca o estabelecimento de um padrão de scholarship produtivo, colaborando com docentes e pares em atividades científicas, que resultam na apresentação e 
publicação de trabalhos, que continuam após a titulação. Já o documento da CAPES estipula a publicação de artigos dos estudantes individualmente ou em parceria com os docentes, o que parece evidenciar a ausência de uma tradição no desenvolvimento da scholarship, quando comparado à tradição dos EEUU. Neste sentido, o documento da AACN considera a inclusão de atividades no curso que preparem os estudantes para assumirem posiçöes de liderança após a titulação. Outro indicador presente nos documentos da AACN e o do CCNEPG considera que os critérios de admissão sejam consistentes com os da instituição.

Os indicadores são consistentes com as características das dimensões formal/técnica e política/sócio-politica, mas como no componente anterior não contempla a dimensão subjetiva, deixando de incluir os sentimentos de satisfação dos estudantes com o sistema educativo e a motivação para comprometer-se e envolver-se politicamente nas decisōes que afetam a profissäo e os interesses da sociedade.

É importante registrar aqui a experiência do Brasil com o doutorado sanduiche, o qual vem ampliando as trocas culturais e educacionais, em nivel nacional e internacionais, favorecendo o intercâmbio entre os cursos e o desenvolvimento de pesquisas colaborativas em temáticas de interesses comuns. Exemplificamos aqui a realização desta modalidade, dentro dos cursos de doutorado do Brasil com países como USA, Canada, França, Suécia e Itália, dentre outros.

\section{AVALIAÇÃO}

Embora não tenhamos incluido o componente avaliação na análise comparativa dos três documentos, consideramos importante tecer alguns comentários. O documento da AACN (1993, p. 3) oferece oito indicadores referentes ao plano de avaliação de qualidade dos cursos de doutorado em enfermagem. Estes indicadores destacam a necessidade de incluir dados (processo e produto), coletados a partir de diversas fontes, internas e externas ao curso, incluindo: estudantes, egressos, professores do curso, empregadores dos titulados, grupos de pares dentro da enfermagem e de órgãos de fomento. O plano de avaliação, o qual deve ser apoiado por recursos humanos, financeiros e institucionais, é sistemático, continuo, amplo, focaliza a missão e os objetivos do curso, e tem aderência aos padrões éticos e processuais da avaliação formal. Estes padrões abrangem confidencialidade e rigorosa análise quantitativa e qualitativa, de forma a possibilitar a comparação do processo e produto do curso aos padrōes da escola/ universidade e de grupos selecionados dentro da enfermagem. Em acréscimo, o plano de avaliação deve, periódicamente, oferecer dados que permitam determinar padrões e tendências e recomendar, futuras direções para o curso, através de feedback para os docentes e admistradores, a fim de promover a melhoria do curso.

Pelo exposto, dá para depreender que a avaliação da qualidade dos cursos de doutorado, nos Estados Unidos, segue um plano sistemático e continuo. Não dispomos de informações mais aprofundadas acerca da periodicidade e da forma como se dá tal avaliação. No entanto, o trabalho de Germain et al. (1994), ao especificar um intervalo de avaliação de 5 a 7 anos contradiz a declaração contida no indicador.

No Brasil, além da avaliação efetuada pela CAPES a cada dois anos, os programas de pós-graduação em enfermagem, em nivel de mestrado e/ou doutorado têm promovido, de forma periódica, a auto-avaliação de seus cursos, contando com a participação de avaliadores extemos nacionais e internacionais. Exemplificamos com as experiências de avaliação ocorridas na UFSC (1986; 1996), na USP-SP (1996) e USP - RP (1997), na UFRJ, cujos resultados foram amplamente divulgados à comunidade cientifica da enfermagem através de publicações.

Também é interessante registrar a experiência de um processo de gestão da qualidade da educação e da pesquisa em enfermagem, realizado durante três anos, com sete Escolas de Enfermagem nacionais, agrupadas na Asociación Venezolana de Educación Superior en Enfermeria - ASOVESE, com consultoria da OPS/OMS - Caracas Esta experiência resultou no 
trabalho de Lopez e Calderón (1996) por nós utilizado como um dos referenciais para avaliação da qualidade.

\section{COMENTÁRIOS FINAIS}

Face ao exposto, fica evidente que o documento da $A A C N$, por ter sido elaborado por enfermeiras, contempla alguns indicadores especificos importantes para o desenvolvimento da disciplina de enfermagem, năo detectados nos outros dois documentos. Por outro lado, alguns indicadores apontados pela CAPES e CCNEPG, não o são no da AACN, embora a partir de nossa experiência saibamos que estes são considerados na vivência profissional das enfermeiras dos EEUU. Esta constatação nos leva a pensar que a tradição no desenvolvimento de cursos de doutorado torna, com o tempo, desnecessários alguns indicadores, na medida em que eles passam a ser incorporados à vida profissional. O mesmo parece não acontecer com os cursos de menor tradição, requerendo, portanto, a especificação destes indicadores.

Conseqüentemente, a tradição de cursos de doutorado em enfermagem consiste em um fator importante a ser considerado, quando se busca padrões locais de indicadores de qualidade, não devendo, por se tratar de especificidades locais, ser contemplados nos padrões globais/ internacionais de indicadores de qualidade.

As especificidades locais/nacionais, detectadas nesta análise, nos permitem identificar tanto forças como fraquezas, no que se refere aos aspectos considerados na avaliação de qualidade dos cursos de doutorado em enfermagem. Podemos destacar como forças para assegurar a qualidade os seguintes pontos: a composição do corpo docente com diversidade de perspectivas intelectuais; a efetivação da interdisciplinaridade, das experiências multi-culturais, e a cooperação local, regional, nacional e internacional. As fraquezas nos remetem para os aspectos relacionados à: dimensão subjetiva da avaliação; reciprocidade na cooperação; desenvolvimento da liderança de docentes e estudantes para posicionarem-se politicamente nas decisões e intervirem na realidade, com base nos interesses da profissão e da sociedade; desenvolvimento de conteúdo curricular e de temáticas de pesquisa que permitam a consolidação da enfermagem como disciplina, encontrando caminhos para melhorar a qualidade de vida em sociedade.

Tanto as forças como as fraquezas estão presentes, de forma mais ou menos acentuada, nos documentos analisados. Exemplificando, podemos citar os documentos dos paises da América Latina, os quais, ao mesmo tempo que têm como força a efetivação da interdisciplinaridade e mostram abertura a um trabalho colaborativo entre disciplinas e cursos no âmbito local, regional, nacional e internacional, também apresentam fraquezas na construção da disciplina de enfermagem. O inverso parece ser verdadeiro quando nos referimos ao documento dos Estados Unidos.

Estas diferentes perspectivas, dentre outras, tem em suas bases a tradição histórica, cultural, política e econômica, as quais levam a uma diversidade de experiências pessoais/ profissionais que, quando compartilhadas globalmente /internacionalmente, poderão enriquecer a cultura e a disciplina de enfermagem, com impactos para a profissão e beneficios significativos para a sociedade.

ABSTRACT: The authors compare /contrast the quality indicators for doctoral programs in three countries, the United States, Brazil and Venezuela, indicating differences and similarities, and identifying issues that can globally affect education in doctoral programs in nursing. Such an analysis is based on three documents: that of the American Association of Colleges of Nursing - AACN (1993); Higher Education Personnel Training Commission- CAPES (1998), and National Consultative Council of Graduate 
Studies of Venezuela - CCNEPG (1987). The authors utilize as a framework the concepts of formal quality and political quality (Demo, 1994) and the sociopolitical, technical and subjective dimensions proposed by López e Calderon (1996). The indicators are distributed among the following components: faculty, program of studies, resources, research and students. Finally, the authors discuss indicators concerning a plan for evaluation of the quality of doctoral programs in nursing.

KEYWORDS: doctoral education, quality assurance, nursing education

RESUMEN:Las autoras comparan/contrastan los indicadores de calidad para programas de doctorado en tres paises: Estados Unidos, Brasil y Venezuela, indicando diferencias y semejanzas y señalando aspectos que puedan globalmente afectar la educación de doctorado en enfermeria. Tal análisis se basa en tres documentos: el de la Asociación Americana de las Escuelas de Enfermería - AACN (1993); el de la CAPES - Brasil (1998); y el del Consejo Nacional Consultivo de Posgrado de Venezuela (1987). Las autoras utilizan como referencial los conceptos de calidad formal y calidad politica (Demo, 1994), y las dimensiones socio-politica, técnica y subjetiva de López y Calderón (1996). Los indicadores se distribuyen entre los siguientes componentes; cuerpo docente, programa de estudios, recursos, investigación y estudiantes. Finalmente, las autoras discuten indicadores respecto al plan para evaluar la calidad de programas de doctorado en enfermeria.

PALABRAS CLAVE: educación en doctorado, garantia de calidad, educación en enfermeria

\section{REFERÊNCIAS BIBLIOGRÁFICAS}

AMERICAN ASSOCIATION OF COLLEGES OF NURSING. Position statement indicators of quality in doctoral programs in nursing, 1993. 4 p. One Dupont Circle, NW, Suite 530. Washington, DC 20036. Mimeogr.

BEARE, Patricia C.: GRAY, Carol J.: PTAK, Helen F. Doctoral curricula in nursing. Nursing Outlook, v.29, n.5, p 311-6, 1981.

CAPES -Ciências da Saúde. Perfil de excelência (Proposta preliminar), 1998 (mimeogr.).

Documento $O$ perfil dos cursos $A$ : critérios a serem adotados pelas Comissőes de Consultores da Capes na avaliaçăo dos cursos de mestrado e doutorado, s/d. (mimeogr).

CARPENTER, Dona Rinaldi. The history of doctoral education in nursing. In: the student on doctoral education in nursing. New York: NLN Press, 1996. p. 1-32.

CONSEJO NACIONAL DE UNIVERSIDADES / CONSEJO CONSULTIVO NACIONAL DE ESTUDIOS PARA GRADUADOS (CCNEPG). Guia para la evaluacion de solicitudes de acreditacion de programas de postgrado - Programa de Doctorado, Caracas, Venezuela, 1987.

DAVIES, Peter (Ed.). American Heritage Dictionary of The English Language. New York, N.Y., 1976.

DEMO, Pedro. Educação e qualidade. Campinas, São Paulo: Papirus, 1994.

DRUGG, Kátia Issa ; ORTIZ, Dayse Domene. O desafio da educação: a qualidade total. Săo Paulo: Makron Books, 1994 
GERMAIN, Carol, P.; DEATRICK, Janet A.; HAGOPIAN, Gloria A ; WHITNEY, Fay W. Evaluation of a PhD program: Paving the way. Nursing Outlook, v.42, n.3, p. 17-122, 1994.

GRACE, Helen K. The development of doctoral education in nursing: in historical perspective. Journal of Nursing Education, v.17, n.4, p. 17-27, 1978.

LÓPEZ, Cira Bracho de; CALDERON. Rutilia. Proceso de Gestion de la Calidad de la Educación y de la Investigacion en Enfermeria. Paper presented at I Congreso Panamericano de Investigación en Enfermeria, Universidad de Carabobo, Valencia - Venezuela, 1996. Mimeogr.

MELEIS, Afaf Ibrahim. Doctoral education in nursing: its present and its future. Journal of Professional Nursing, v.4, n.6, p436-446, 1988.

MURPHY, Juanita F. Doctoral education in, of, and for nursing: na historical analysis. Nursing Outlook, p. 645-649, 1981

SNYDER-HALPERN, Rita. Nursing doctorates: is there a difference? Nursing Outlook, v.34, n.6, p. 284-5, 291, 1986.

UNIVERSIDADE FEDERAL DE SANTA CATARINA. Anais dez anos de pós-graduação em enfermagem: avaliação e perspectivas. Florianópolis, SC: Editora UFSC, 1986.

UNIVERSIDADE FEDERAL DE SANTA CATARINA Avaliação Interna da Pós Graduação em Enfermagem da UFSC. Revista Texto \& Contexto, v. 5, 1996.240 p. Número especial.

Recebido em julho de 2000

Aprovado em agosto de 2000 